\title{
Tau peptide signals are seen in Parkinson subjects by Broderick Probe ${ }^{\circledR}$ sensors
}

\begin{abstract}
This "short but sweet" clinical paper is about a next generation "uptick" nanotechnology that demonstrates a unique, real time imaging inventive art enabling a different kind of look at the brain, actually, to see inside neuronal and glial circuitry in the brain and spinal cord of the living human being and animal. "Lewy body dementia is characterized by the abnormal buildup of proteins into masses known as Lewy bodies. This protein is also associated with Parkinson's disease. People who have Lewy bodies in their brains also have the plaques and tangles associated with Alzheimer's disease. Apr 26, 2019, Mayo clinic". " This is the problem and thus far, the problem is addressed primarily after autopsy, called post mortem, also problematic. Therefore, the purpose of this paper is to introduce the online and real time and spatial sensitive voltaic image of the Tau peptide complex video-tracked by the BRODERICK PROBE ${ }^{\circledR}$ biomedical sensors in striatum of the living Parkinson subject. This nanoprobe enables studies of the striking consequences among intensities of phosphorylated Tau. Identifying Tau on line is unmistakably relevant to longevity, both individual and societal and this relevance is inexorably critical to and on behalf of humanity per se.
\end{abstract}

Keywords: Parkinson, Alzheimer, disease, epilepsy, dementia, Lewy, cognition, sensors, device, surgery, nanotechnology, pharmaceutical, aging, isoform, unphosphorylated, phosphorylated, hyperphosphorylated, protein, unfolding, aggregation, Tau, telomere, DNA, RNA, mRNA, amyloid (A $\beta)$, protein kinase, prion, alpha $(\alpha)$ synuclein, Positron Emission Tomography (PET), Nuclear Magnetic Imaging (NMR)
Volume II Issue 2 - 202 I

\section{Patricia A Broderick}

Department of Molecular, Cellular, and Biomedical Sciences, City University of New York (CUNY) School of Medicine, USA

\author{
Correspondence: Dr. Patricia A Broderick, Department of \\ Molecular, Cellular, and Biomedical Sciences, City University of \\ New York (CUNY) School of Medicine, USA,
}

Emailbroderick@med.cuny.edu

Received: March 30, 2021 | Published: April 22, 2021

\section{Introduction}

About this next generation "uptick" nanotechnology, it is further specified as Neuromolecular Imaging, abbreviated NMI. The BRODERICK PROBE ${ }^{\circledR}$ biomedical library of sensors are the principal part of this LIVE and online novel imaging nanotechnology. The original formulae of these sensors are comprised of carbon/ lipid/phosphotidyl polymers and this dramatic advance has transformed spectrometry into a spectral analysis in the form of LIVE electrochemistry using carbon allotropes in lipid matrices to videotrack time sensitive movement of neuromolecules while at the same time, the sensors image from movement nuclei and further while the subject is actually walking and/or in altered states of consciousness and further to another Broderick sensing device, a semiconductor carbon allotrope, fullerene and further to a photo luminescent fiber optic protein probe. Each and every sensor is inventive art formalized under the umbrella of the BRODERICK PROBE ${ }^{\circledR}$ trademark.

When we look at the diagnosis of Parkinson and Alzheimer disease, the conventional empirical and theoretical art virtually remains at a loss for adequate treatment given the caveat that positron emission tomography (PET), images Tau and $\mathrm{A} \beta$ plaques to assess cognitive deficits. Nonetheless, when we discern Parkinson disease, Lewy Body Dementia (LBD) and Alzheimer disease including other neurodegenerative disorders of asynchrony similar to epileptogenic seizures, we are met with a conundrum to either diagnose or treat because central distinctions among these deadly diseases are beset with formerly known exact criteria but these criteria are now confused. In fact, we thought we had this down to a dance step as the Lewy body seemed to be a suitable solution for a comfortable distinction. As Lewy bodies so true of Parkinsonian patients and animals are comprised of alpha $(\alpha)$ synuclein, we were able to say in the past that
Parkinsonian subjects present with Lewy bodies, Alzheimer subjects present with $\mathrm{A} \beta$ plaques plus Tau and epilepsy subjects exhibit Tau.

What has happened? These essentially untreatable diseases present with horrendous complexities such that Parkinsonian patients now present with Lewy bodies plus $\mathrm{A} \beta$ plaques and Tau; Alzheimer subjects present with A $\beta$ plaques, plus Lewy bodies plus Tau. To compound the felony, the trio of deadly diseases come from within, infections from within, a type of protein unfolding, derived from prions, called prionopathies and derived from alpha synuclein, called synucleinopathies. To this point, post mortem studies have assayed Lewy bodies, alpha synuclein, the amyloid precursor proteins and Tau but the studies are performed post mortem which means after death and the limitations of "life after death" may simply add to already mis-diagnoses.

Tau is a group of six highly soluble protein isoforms produced by alternative splicing from the gene MAPT (microtubule-associated protein tau). Microtubules are tubular polymers, comprised of tubulin that self-assemble on a scaffold-like design to make up the infrastructure of a cell; these tubular polymers act as a kind of railway to transport organelles throughout the cytoplasm. These intracellular structures have a stable region, as well as a region that remains dynamic, both important to playing their role in a cell. It is noteworthy that the conventional role of Tau resided in the maintenance of microtubule stability in axons, an established thesis that is highly questioned in current times. ${ }^{2-5}$ Tau peptides and proteins are abundant in the neurons of the central nervous system (CNS). It is generally known that Tau is less common elsewhere but are also expressed at very low levels in CNS astrocytes and oligodendrocytes. As defined herein, Tau protein/ peptide includes but is not limited to phosphorylated forms. Tau is a healthy protein! It is healthy until it is unhealthy by dysregulation 


\section{likely, via hyper phosphorylation and/or isoform mechanisms.}

Thus, Tau, when unhealthy, is the biomarker for Tau pathologies or tauopathies. Tauopathies are neurodegenerative disorders characterized by the deposition of abnormal Tau in the brain. Pathologies and dementias of the nervous system such as, for example, Alzheimer disease, Parkinson disease and Tau-associated Epilepsy are associated with Tau that have become hyperphosphorylated insoluble aggregates called neurofibrillary tangles. The path from phosphorylation to hyperphosphorylation to aggregation is puzzling; this is why the BRODERICK PROBE $^{\circledR}$ polymer is important. The nanoprobe acts to image through mechanisms of phosphorylation, enabling precise images showing differential degrees of phosphorylation. Therefore, we can study degrees of phosphorylation to the point at which aggregation occurs, the proverbial "hitting the nail on the head". The BRODERICK PROBE ${ }^{\circledR}$ enables the online view, that is, actually the ability to see the point at which there is little or no return from Tau phosphorylation as it progresses to aggregation online. Such a feat then allows the view of the image of the Tau peptide complex and moreover, the image of the selective Tauopathies.

Therefore, the purpose of this paper is to introduce the online and real time voltaic image of the Tau peptides by the BRODERICK PROBE ${ }^{\circledR}$ biomedical sensors in the striatum of the living Parkinsonian animal model. Identifying Tau on line brings real time diagnostic, therapeutic and prognostic answers to a world that is daily increasing in cases of dementia not at all material to its derivation. The quest is imperative as the consequences to not pursuing this line of "research questions" may be our proverbial "Waterloo". Indeed, this unwise alternative may well be the demise of a cognitive human race at least as it is recognized presently as a kingdom domain, inclusive of separate and distinct species of plants and animals ${ }^{6}$ albeit there are now six kingdoms classified. Humans are in the animal kingdom. ${ }^{7}$

\section{Methods}

The BRODERICK PROBE ${ }^{\circledR}$ series of biosensors work by electrochemical detection and are comprised of carbon and/or carbon allotropes in one example and fiber optic proteins in another of the many examples. In the present study, the original carbon sensors, the carbon/lipid/phosphotidyl polymers were used. This biomedical device is unique from other techniques and methodologies in that this series of biosensors can image signals in vivo, in vitro or in-situ within sub-seconds in real time and the sensors are so tiny that they can be used anywhere and for any length of time and controlled studies have found these operationally stable biomedical biosensors to be void of causing gliosis (scar tissue) or producing infection (bacterial growth).

Further details and examples of Broderick probes may be found ${ }^{8-15}$ all to P. A. Broderick including circuit diagrams and methods of making Broderick probes. Broderick probes may be in electrical contact with an auxiliary electrode and/or a reference electrode. It will be apparent to those of ordinary skill in the art, particularly in view of the cited patent documents, that "Broderick probe" is a term that relates to a number of electrodes that vary by composition and the type of circuit in which it is employed and that these variations give rise to differences in detection properties.

This inventive art relates to the recent leaps in areas not only in spectroscopy but in microscopy and this inventive art gives scientists a nanotechnology that takes advantage of LIVE phenomena that occur naturally in the brain and spinal cord where matter is organized at the nanoscale. The science of advanced nanomaterials allows these phenomena to be studied based on quantum mechanics and allows focus on an expanded surface area. Indeed, the majority of biological processes do occur at the nanoscale, bringing us to brand new constructs and designs and the like in medicine, imaging, computing, printing, chemical catalysis, electrocatalysis and materials synthesis. We cannot think of "Nanotechnology" as just working at ever smaller dimensions. This would be a mistake.

Rather, imagine working at the nanoscale that engages scientists in a brave new world, talking with each other to utilize unique platforms to cure disease. Imagine uniting diagnostic and therapeutic platforms such as physical, biochemical, chemical, mechanical, electrical, piezoelectric, photoelectric, stimulating and optical properties that naturally occur at nanoscale. The BRODERICK PROBE ${ }^{\circledR}$ works at the nanoscale, enabling tunability of carbon allotropes and opsin laser photodiodes that see inside the brain without opening or minimally touching the brain as well as semiconductors such as fullerene to fulfill the hope of a better quality of life beyond degeneration. In this clinical report, the original carbon allotropic polymer expands the realm of the uses of the BRODERICK PROBE ${ }^{\circledR}$ to advance the quest for diagnoses and treatment of Tau-related physiologies and pathologies in the healthy and the unhealthy living subject.

Advantages over other methodologies are clear. The literature cites that the dialysis (microdialysis) method has disadvantages relative to sampling time resolution. For one example, dialysis offers somewhat limited time resolution. The use of highly sensitive analytical techniques (e.g. neuromolecular imaging with the Broderick probe) images in temporal resolutions of seconds and less, spatial resolutions of nanospheres and current resolution of nanoamperes. Dialysis samples may not provide real time information regarding changes in the neurochemical environment. Also, dialysis is an invasive technique and therefore causes some damage to the tissue under study and critically, the dialysis process creates an area around the membrane in which all solutes capable of crossing the membrane are depleted. ${ }^{16}$

Postmortem studies are daily used to distinguish between Alzheimer disease and the age-old expression of "dementia"as these diseases relate to the pathological presence of $A \beta$ plaques and neurofibrillary tangles versus Lewy bodies respectively. Tau and A $\beta$ plaques are seen as a double prion disease post mortem by Prusiner who discovered the prion. ${ }^{17,18}$ Alzheimer patients are often seen post mortem to exhibit alpha synucleated Lewy bodies in addition to $\mathrm{A} \beta$ plaques, thus resulting in confusion, compounded with the innate hindrance of studying any disease post mortem. Physiological and pathological processes may not be translatable to life per definitionem. The BRODERICK PROBE ${ }^{\circledR}$ provides solutions since Tau is imaged LIVE. NMI of Tau's anionic redox dynamic movement is likely away from the microtubule and is comprised likely in a metal complex. Metal complexes of Tau are described by Gorantla et. al,. ${ }^{19}$ Detachment from the microtubule assists in seeing Tau as a neuroprotector. ${ }^{20}$

Imaging the neurotransmitter facets of the disease called, Parkinsonian, is a nanotechnology and a nanobiotechnology which is studied extensively in the Broderick laboratory. The laboratory is well known for delineating the neuroprotective transmitter elements of the disease as well as its degenerative elements in the living animal model of this movement and cognitive disorder, named after Parkinson. Often beset with depression and consequent to treatment, per se, to psychosis, the disease is a Lewy body disease of alpha $(\alpha)$ synuclein; the disease is a prionopathy and a synucleinopathy. Details of the experimental design are provided..$^{21,22}$

The precision of this nanoprobe is enhanced further beyond the previously used techniques because the molecular transmission imaging is derived from the actual basal ganglia neurons responsible 
for motor function. The BRODERICK PROBE ${ }^{\circledR}$ sensed striatal neurons in order to study the functional activity of efferent dorsal striatal neuronal circuitry from the substantia nigra pars compacta (SNPC). Parkinson model subjects were lesioned at the SNPC using 6-hydroxydopamine in order to inhibit neurons in the dorsal striatal area originating from the SNPC from producing dopamine; surgical technicians at the North Carolina facility, the International Charles River Laboratories, performed the expert lesion technology.

\section{Results}

The neuroprobe/nanosensor signals from the brain are remembered in the case of the original polymer, the carbon polymer, the carbon/ lipid/phosphotidyl polymer.

The following drawings are provided by way of example only and without limitation, wherein like reference numerals (when used) indicate corresponding elements throughout the several views. The reference numerals are described in detail within the patents.

Figure 1 shows the operation of a Broderick probe in detecting neurotransmitters and a scan resulting there from.

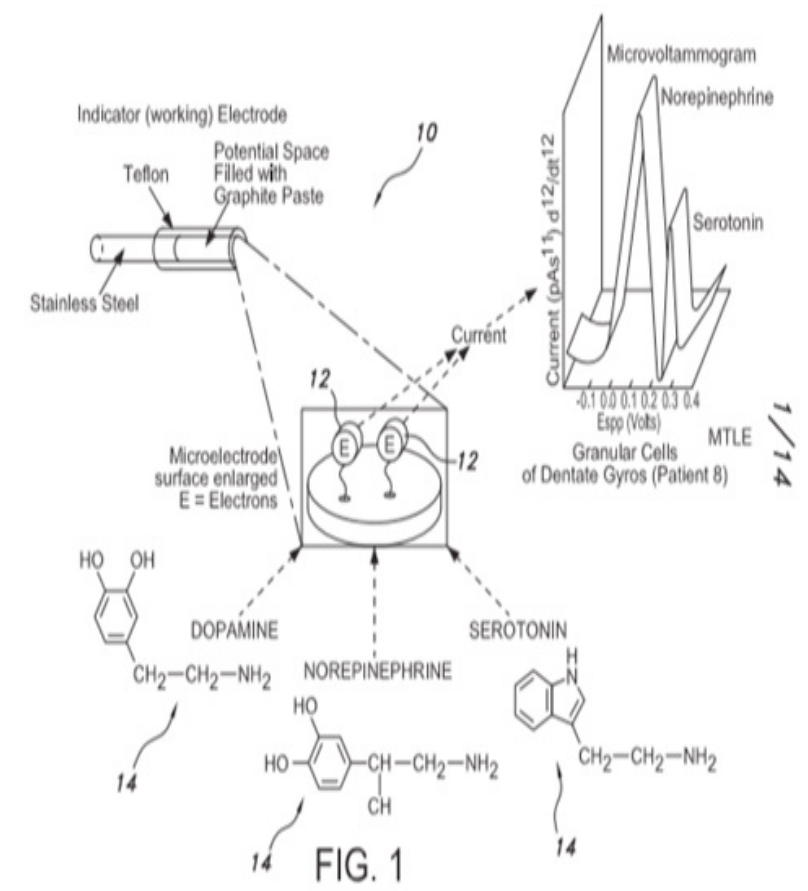

Figure I Norepinephrine (NE) and serotonin (5-HT) from the granular cells of the dentate gyrus in an epilepsy patient are shown in the upper right quadrant.

Figure 2 The Photoluminescent Fiber Optic Protein Probe. A schematic of the opsin, rhodopsin is shown to the left. The Broderick Semiconductor Fullerene Probe is shown to the right in the schematic as encased in the Broderick probe original carbon/lipid/phosphotidyl polymer. Each of these sensing devices are trademarked, the BRODERICK PROBE ${ }^{\circledR}$.

Figure 3 shows the sensing devices operating within the BRODERICK PROBE ${ }^{\circledR}$ photoluminescent fiber optic protein probe as the sensing device interacts with the lobes of the brain that connect via the radio frequency light spectrum. Although Ultraviolet Light is shown as an example, the patents do not limit the photons of light chosen. However, the light selected for the experimental design is preferably Near Infrared, it is not limited to the Near Infrared
Spectrum that the author has chosen for the Tau epilepsy patients and the patients presenting with Alzheimer disease and/or the disorder of Parkinson. In the bottom left, the application of piezoelectric sensing devices is shown as proposed and patented to act in concert with the BRODERICK PROBE ${ }^{\circledR}$. Data output derived from the calcium channel blocker, nimodipine, is shown to the middle right. Same subject design is an advantage of our nanotechnology, NMI. The data were gleaned from a hypoxic-hypoxia animal model designed in our laboratory to introduce the mechanism of action of memory defects that commonly occur in post-operative surgery. ${ }^{23}$

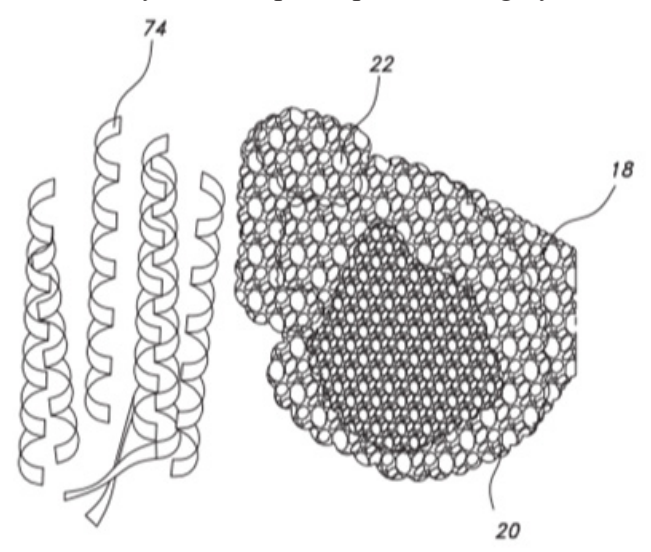

Figure 2 The Photoluminescent Fiber Optic Protein Probe. A schematic of the opsin, rhodopsin is shown to the left. The Broderick Semiconductor Fullerene Probe is shown to the right in the schematic as encased in the Broderick probe original carbon/lipid/phosphotidyl polymer. Each of these sensing devices are trademarked, the BRODERICK PROBE ${ }^{\circledR}$.

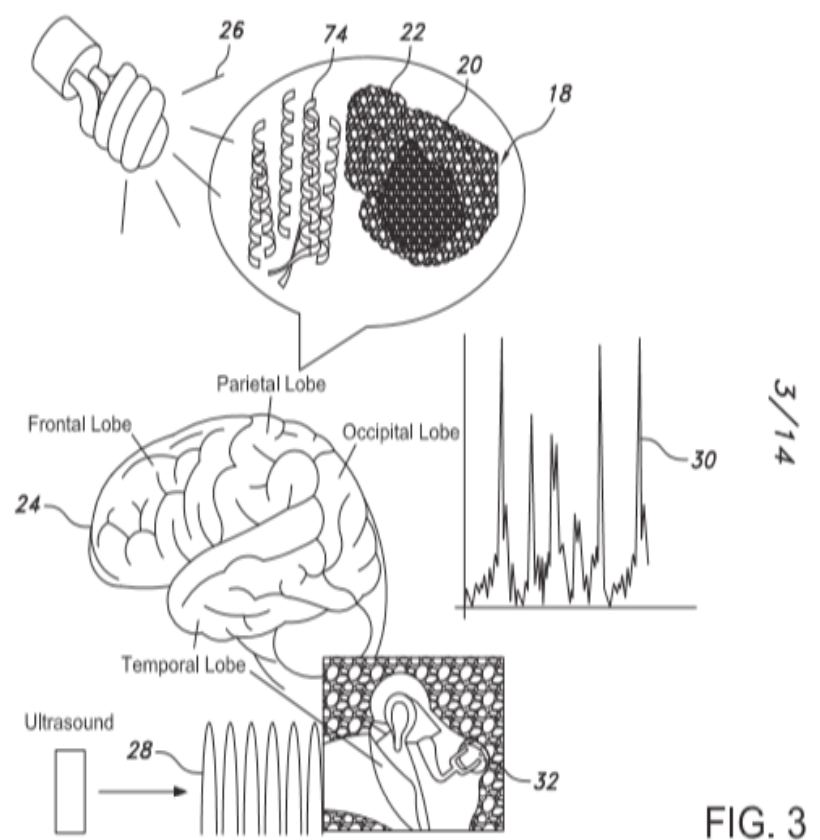

Figure 3 Shows the sensing devices operating within the BRODERICK $\mathrm{PROBE}^{\circledR}$.

Figure 4 shows, using the BRODERICK PROBE $^{\circledR}$ lightly touching the striatum in a substantia nigra pars compacta lesioned Parkinson's [PD] animal subject, in real time and online endogenous baseline neurochemical profile hitherto not previously accomplished. Serotonin (5-HT), L-tryptophan (L-Trp) and neuropeptides (dynorphin and somatostatin) were present. Note that dopamine 
(DA) neurotransmitter was absent in lesioned group (PD). In fact, the neurotransmitter, serotonin, is absent at times, especially when there is corresponding motor incoordination as is the case with degenerative disorders, specifically, the disease of Parkinson in this example. The direct cause and effect discovery between release of a serotonin molecule with a specific classified functional movement as it happens in real time,is critical to the meaning of the data presented since Temporal Synchrony, shown between serotonin and movement, may well define the healthy brain. ${ }^{24}$ The importance of such real time correlations between brain and behavior has reached the textbook. ${ }^{25}$

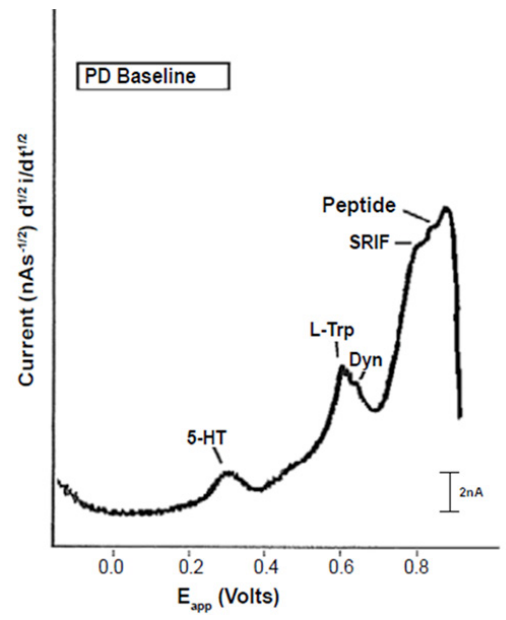

Figure $4 \mathrm{NMI}$ results from lesioned group (PD animal model) with endogenous neurochemical profile. On the $x$-axis: oxidation potential in term of applied voltage in volts (V). On the $y$-axis: current in nanoamperes (nA). NMI was conducted using the BRODERICK PROBE ${ }^{\circledR}$ laurate biosensor. Serotonin (5-HT), L-tryptophan (L-Trp) and neuropeptides (dynorphin and somstostatin) were present. Note that dopamine (DA) neurotransmitter was absent in lesioned group (PD).

Figure 4 The signal marked "peptide" is the Tau-associated peptide/protein. This is the first report of Tau signaling LIVE in electroactive images in a LIVE subject presenting with and exhibiting the cardinal symptomatology of the brain disease called Parkinson. In one embodiment, the electrode or sensor or nanoprobe detects the activities of Tau proteins/peptides (i.e., "Tau"). ${ }^{14}$

\section{Discussion}

Tauopathies are a group of neurodegenerative diseases in which aggregates of the hyperphosphorylated form of the microtubuleassociated protein (MAP), tau, are believed to play a critical role in the pathogenesis. Many of the tauopathies are associated with an increased incidence of development of epilepsy. Deposits of hyperphosphorylated tau have also been documented pathologically in animal models of epilepsy, and in patients with drug-resistant focal epilepsy who have undergone epilepsy surgery. The precise mechanisms by which hyperphosphorylated tau contributes to epileptogenesis are currently uncertain, but there is increasing evidence from animal models that targeting tau-based mechanism, either genetically or pharmacologically, can have both antiseizure and antiepileptogenic effects. Indeed, animal models of epilepsyassociated tauopathies, biomarkers of tau pathology, experimental evidence implicating hyperphosphorylated tau in the pathophysiology of acquired epilepsies, in addition to therapies for Tau epilepsies are described in ${ }^{26}$ and critical sensing epilepsy work is contained in..$^{27,28}$

The Tau gene and its reported six Tau isoforms have been found in the brain of Alzheimer's patients. ${ }^{29}$ In Tau-related prionopathies, for example but not limited to mad cow, people and or patients who are affected by this disease experience unusual sensations that include prickling or tingling feelings in the face, hands, legs, and feet while sometimes sores appear on the feet for example. Sensations on these body parts can transform into burning sensations and permeate other body parts in a "pins and needles" sensation. Paresthesia can occur, causing a persistent lack of feeling in these body parts, producing clinical numbness.

\section{Dugger et al., ${ }^{30}$ provides data that}

\section{QUOTE}

"dementia is a frequent complication of Parkinson's disease (PD) and that about half of PD dementia (PDD) is hypothesized to be due to progression of the underlying Lewy body pathology into limbic regions and the cerebral cortex while the other half is thought to be due to coexistent Alzheimer disease (AD). Clinically, however, these are indistinguishable. Since the spread of amyloid plaques to the striatum has been reported to be a sensitive and specific indicator of dementia due to $\mathrm{AD}$, these authors analyzed neuropathologically-confirmed cases of PD without dementia (PDND, N=31), PDD without AD $(\mathrm{PDD}, \mathrm{N}=31)$ and $\mathrm{PD}$ with dementia meeting clinicopathological criteria for AD (PDAD, $\mathrm{N}=40$ ). The minimum diagnostic criterion for $\mathrm{AD}$ was defined as including a clinical history of dementia, moderate or frequent CERAD cortical neuritic plaque density and Braak neurofibrillary stage III-VI. Striatal amyloid plaque densities were determined using Campbell-Switzer and Thioflavine S stains. Striatal plaque densities were significantly higher in PDAD compared to PDD $(p<0.001)$. The presence of striatal plaques was approximately $80 \%$ sensitive and $80 \%$ specific for predicting $\mathrm{AD}$. In comparison, the presence of cerebral cortex plaques alone was highly sensitive (100\%) but had poor specificity ( $48 \%$ to $55 \%$ ). The results suggest that striatal amyloid imaging may be clinically useful for making the distinction between PDD and PDAD."

The Dugger et.al, work is an important contribution to distinguishing Parkinsonism by amyloid concentration. Positron Emission Tomography in cerebrospinal fluid is new and hopeful. ${ }^{5}$ Nuclear magnetic resonance (NMR) for flexible Tau forms and CryoEM for the most rigid forms or fluid immuno-diagnosis coupled to brain imaging research such as the BRODERICK PROBE ${ }^{\circledR}$ bring promise. Video microscopy enables another useful tool in this highly active and necessary research arena, both clinical and pre-clinical. Antibody labeling is daily used antemortem and post mortem; it has brought forth valuable data on tauopathies despite its post mortem status. Telomere length on the ends of strands of deoxyribose nucleic acid (DNA) is associated with deciphering correlative cognitive enhancement or decline, another avenue of strategic cures for tauopathies. ${ }^{31}$

Finally, in press, from Singapore, is the author's authentic imaging textbook which explains with original experimental protocols in detail, the know-how of NMI along with describing the discoveries made by the BRODERICK PROBE ${ }^{\circledR} 32$

\section{Conclusion}

However, it is the Broderick Laboratory who identifies herein, the LIVE electroactive image for Tau in the precise striatal basal neurons of the living subject as a metal complex phosphorylated, dynamic and presenting with Parkinsonian disease in this clinical paper. Mueller et al., ${ }^{5}$ posit: "tau functions as a signaling hub, regulating a wide set of cellular processes through the modulation of selected kinases and phosphatases." Thus, the elusive nature of Tau is no longer elusive as Tau is showcased herein as a dynamic redox reaction occurring LIVE in the brain of the living Tau patient and animal via the phosphorylated BRODERICK PROBE ${ }^{\circledR}$. 


\section{Acknowledgments \& funding}

The author wishes to thank the Broderick Brain Foundation, the F.M. Kirby Foundation, the Center for Advanced Technology, CUNY, and the MacKenzie Foundation for partial support of our laboratory and students during these studies. It is important to note that this body of work, the development and pioneering of Neuromolecular Imaging and the BRODERICK PROBE ${ }^{\circledR}$ has taken place diligently, enduring reliability for many years. Other grants including the National Institute of Health, National Institute on Drug Abuse, The Lowenstein Foundation, the FACES and PACE Foundations for Epilepsy and The Upjohn Pharmacia Company in Michigan (Arvid Carlsson, MD and Monty Piercey, PhD, in memoriam) and the NYU Langone Depts of Neurology and Comprehensive Epilepsy Center in addition to the NYU Langone Dept. of Anesthesiology deserve honorable mention. Finally, Eazysense Nanotechnologies, Inc. is marketing this intriguing nanosensor, the BRODERICK PROBE ${ }^{\circledR}$ library of miniature biomedical sensors and devices.

\section{Conflicts of interest}

The author declares no conflict of interest.

\section{References}

1. Alzheimer's disease. Symptoms and causes. Mayo Clinic. 2020.

2. Trinczek B, Biernat J, Baumann K, et al. Domains of tau protein, differential phosphorylation, and dynamic instability of microtubules. Molecular biology of the cell. 1995;6(12):1887-1902.

3. Fichou Y, Al-Hilaly YK, Devred F, et al. The elusive tau molecular structures: can we translate the recent breakthroughs into new targets for intervention? Acta Neuropathol Commun. 2019;(7)31.

4. Scholl M, Maass A, Mattson N, et al. Biomarkers for tau pathology. Mol Cell Neurosci. 2019;97:18-33.

5. Mueller RL. Tau: A Signaling Hub Protein. Frontiers. 2021.

6. Linnaeus C. Systemanaturae, sive regna trianaturaesystematic eproposita per classes. ordines, genera, \& species. Leiden: Haak. 1735; p. 1-12.

7. Bailey RB. The Six Kingdoms of Life. Thought Co. 2020.

8. Broderick PA. Cathodic Electrochemical Current Arrangement with Telemetric Application. US Patent Serial No. 4, 883,057. 1989.

9. Broderick PA. Microelectrodes and their use in Cathodic Electrochemical Current Arrangement with Telemetric Application. US Patent Serial No. 5,433,710. 1995 .

10. Broderick PA. Microelectrodes and their use in an Electrochemical Arrangement with Telemetric Application. US Patent Serial No. 5,938,903, Issued. 1999.

11. Broderick PA. Noninvasive Electroactive Photonic Sensor with Polymer Memory Transduction Using Organic and Inorganic Elements as Platforms. US Provisional Application No. 62/273,693. 2017.

12. Broderick PA. Noninvasive Electroactive Photonic Protein Sensor with Polymer Photovoltaic Optics for Memory Transduction Using Organic and Inorganic Elements as Platforms. US Non-Provisional International Application No. PCT/US 16/68879. 2020

13. Broderick PA. Identification, Diagnosis, and Treatment of Neuropathologies, Neurotoxicities, Tumors, and Brain and Spinal Cord Injuries Using Electrodes with Microvoltammetry. US Patent Serial No. 10,980,460, Issued. April 20 2021.

14. Broderick, P.A. Identification, Diagnosis, and Treatment of TauAssociated Pathologies Using Electrodes. US Provisional Application No. 63/130,453, December 24th, 2020.
15. Broderick PA, Pacia SV. Identification, Diagnosis, and Treatment of Neuropathologies, Neurotoxicities, Tumors, and Brain and Spinal Cord injuries using Microelectrodes with Microvoltammetry. US. Patent Serial No. 7,112,319. 2006

16. Chefer VI, Thompson AC, Zapata A, et al. Overview of brain microdialysis. Curr Protoc Neurosci. 2009; Chapter 7:Unit7.1.

17. Prusiner SB. Nobel Lecture: Prions. Proceedings of the National Academy of Sciences. 1998;95(23):13363-13383.

18. Prusiner SB, Woerman AL, Mordes DA et al. Evidence for $\alpha$-synuclein prions causing multiple system atrophy in humans with parkinsonism. Proceedings of the National Academy of Sciences of the United States of America. 2015;112(38): E5308-E5317.

19. Gorantla NV, Landge VG, Nagaraju PG, et. al, Molecular Cobalt (II) Complexes for Tau Polymerization in Alzheimer's Disease. ACS omega. 2019;4(16):16702-16714.

20. Schneider A, Biernat $\mathrm{J}$, von Bergen $\mathrm{M}$, et Al. Phosphorylation that detaches tau protein from microtubules (Ser262, Ser214) also protects it against aggregation into Alzheimer paired helical filaments. Biochemistry. 1999;38(12):3549-3558.

21. Broderick PA, Kolodny EH. Real Time Imaging of Biomarkers in the Parkinson's Brain Using Mini-Implantable Biosensors. II. Pharmaceutical Therapy with Bromocriptine. Pharmaceuticals (Basel, Switzerland). 2009;2(3):236-249.

22. Broderick PA, Wenning L, Li YS. Neuromolecular imaging, a nanobiotechnology for Parkinson's disease: advancing pharmacotherapy for personalized medicine. Journal of Neural Transmission (Vienna, Austria). 2016;201;124(1):57-78.

23. Haile M, Broderick P, Li Y, et al. Nimodipine Reverses the Elevation of Synaptic Striatal Dopamine and Serotonin During In Vivo Hypoxia. The Open Neuroscience Journal. 2011;5: 1-7.

24. Broderick PA, Neuromolecular Imaging Shows Temporal Synchrony Patterns between Serotonin and Movement within Neuronal Motor Circuits in the Brain. Brain Sci. 2013;3( 2):992-1012.

25. Broderick PA, Wenning L. Neuromolecular imaging in Parkinson's disease. In: Martin CR, Preedy VR. Editors. Genetics, neurology, behavior, and diet in parkinson's disease, The Neuroscience of Parkinson's Disease, Elsevier Academic Press, United Kingdom Chapter. 2020;(2):16:251-265

26. Zheng, P, Shultz S, Hovens C, et al. Animal Models of Acquired Epilepsy and Tauopathies. Models of Seizures and Epilepsy. 2017;1031-1041.

27. Broderick PA. Sensing the epilepsy brain: a unique nanosurgical biomedical device to treat the epilepsy patient. MOJ Clin Med Case Rep. 2018;8(2):60-65.

28. Broderick PA, Leslie Wenning. Neuromolecular Imaging and BRODERICK PROBE nanobiosensors reveal a temporal synchrony in brain rhythms in neural transmission online with movement designs during natural physiology: Temporal asynchrony is imaged online in the same subject during pathology, Medical Research Archives, 2018;6(7):118.

29. Noble W, Hanger DP, Miller C, et. al, The importance of tau phosphorylation for neurodegenerative diseases. Frontiers in Neurology. 2013;4:83.

30. Dugger BN, Serrano GE, Sue LI, et al. Presence of striatal amyloid plaques in Parkinson's disease dementia predicts concomitant Alzheimer's disease: Usefulness for amyloid imaging. Journal of Parkinson's disease. 2012;2(1):57-65.

31. Broderick PA. NEUROIMAGING: Sensing Biochemistry in the Brain, 2021. Jenny Stanford Publishing Pte Ltd., Singapore.

32. Mahoney ER, Dumitrescu L, Seto M, et al. Telomere length associations with cognition depend on Alzheimer's disease biomarkers. Alzheimer's \& dementia. 2019;5(1):883-890. 